\title{
Study the Specifications of the Thread through the Construction of the Pipe on the Ring Spinning Machine
}

\author{
Mohamad Yaser Joha', Easa Morad ${ }^{1,2}$ \\ ${ }^{1}$ Postgraduate Student (Msc.), Department of Mechanical Engineering of Textile Industries and Their \\ Technologies, Faculty of Mechanical and Electrical Engineering, Damascus University, Damascus, Syria \\ ${ }^{2}$ Ass. Prof. Mechanical Department, Faculty of Mechanical and Electrical Engineering, Damascus University, \\ Damascus, Syria \\ Email: yaserjo98@gmail.com
}

Received 14 December 2014; accepted 20 April 2015; published 23 April 2015

Copyright (C) 2015 by authors and Scientific Research Publishing Inc.

This work is licensed under the Creative Commons Attribution International License (CC BY).

http://creativecommons.org/licenses/by/4.0/

(c) (i) Open Access

\begin{abstract}
In ring spinning, tension develops in the yarn mainly because, to move the Traveler and the balloon length, $L b$, around the spindle axis, and to wind the yarn onto the spinning bobbin, work must be done against the frictional force of the ring on the Traveler and of the Traveler on the yarn, as well as against the air drag on the Traveler and on the balloon length. This work is additional to that needed to overcome the friction of the spindle bearings and the air drag on the forming yarn package. The tensions in the yarn during ring spinning may be considered with respect to three zones: 1) the yarn formation zone (i.e., the zone between the pigtail lappet guide and the front rollers of the drafting system); 2) the winding zone (i.e., the zone between the Traveler and the bobbin forming zone); 3 ) the balloon zone (i.e., the zone between the Traveler and lappet guide), where the yarn tension changes noticeable shape by relation with Winding Qatar (the yarn tension increases whenever Winding Qatar reduces).
\end{abstract}

\section{Keywords}

The Yarn Formation Zone, The Winding Zone, The Balloon Zone

\section{Introduction}

In ring spinning the Traveler circulates the ring; it pulls with it. The yarn length is between the yarn guide and the traveler. This length circulates the axis common to the spindle, ring, and lappet guide. In winding, discussed in, over-end withdrawal is used to pull the yarn off the ring spinning bobbin in the direction of the bobbin axis, 
and the yarn passes through a pigtail guide on the axis. At withdrawal speeds of up to $25 \mathrm{~m} / \mathrm{min}$, the yarn tends to follow a direct path from the unwinding point on the package to the guide. In this situation, the yarn tension is almost entirely the result of frictional drag on the package surface. Winding speeds are very much higher than $25 \mathrm{~m} / \mathrm{min}$. At these higher speeds, the yarn balloons and, similar to ring spinning, the balloon geometry is determined by the equilibrium of the above-mentioned forces. Yarn ballooning is a physical phenomenon of practical interest. It sets the minimum distance of separation that must occur between spindle positions on a ring spinning machine so as to prevent adjacent balloons from colliding. Stable ballooning is essential for reduced machine stoppages. Balloon stability governs the balloon height/ring diameter relationship and thereby the package size. These factors, in turn, influence the production rate, energy cost, and (in certain cases) fabric quality [1].

\section{Ring Spinning}

The objectives of ring spinning are:

- to thin out the roving and give it the desired count;

- to impart a specific twist to the yarn so as to give the yarn the desired resistance;

- to collect the yarn into a package (i.e. the bobbin) for simpler storage and handling.

The Working Principle of the Ring Spinning Frame

The ring spinning frame operates as follows (Figure 1):

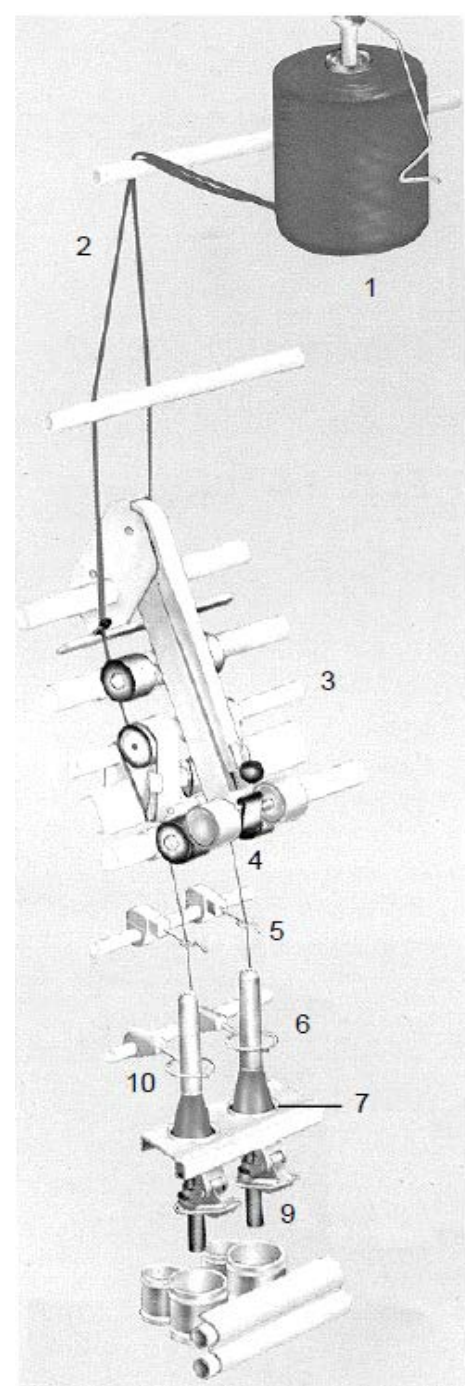

Figure 1. Ring spinning diagram. 
1) the bobbins (.1.), coming from the finisher rubbing frame, are suspended on the feeding rack above the spinning frame- - one bobbin for each two spindles;

2) the roving (.2.), unwound tangentially from the bobbins, are pulled by the feeding cylinders of the drafting unit (.3.) where they are thinned out;

3) once the fibers leave the exit rollers of the draw frame, the forming yarn (.4.), pulled by the revolving spindle, passes through the yarn guide (.5.), inside the anti-balloon ring (.6.) and inside the Traveler (.7.), and then is twisted and wound on the tube (.8.) placed on the spindle (.9.). The yarn twists since when winding on the small tube on the rotating spindle; it makes the Traveler (.7.) rotate around the ring (.10.), with a movement concentric to the spindle (Figure 1); at each turn of the Traveler around the ring, the yarn makes a twist in the segment of yarn between the drafting cylinders and the Traveler. The winding of the yarn on the tube can be achieved since the Traveler movement is helped and driven by the yarn; the rotation speed of the Traveler is lower than the tube (spindle) speed due to the frictional force generated when sliding on the ring (and, to some extent, also to the resistance of the air to the motion of the yarn between the yarn guide and the Traveler).

The distribution of the yarn on the tube, allowing the formation of the bobbin, takes place by means of the alternated vertical stroke of the rings, arranged on a horizontal rail; the stroke reversal points are not fixed but move continuously upward. The travel of the rail is always steady; the rail begins to move near the base of the tube and stops after reaching (through the continuous upward shift of its stroke reversal points) the top edge of the tube.

a) Roving feeding: The roving feeding system, though being a quite simple device, can greatly affect the number of defects of the yarn; in particular, if the roving unwinds incorrectly, possible, cuts, or even breakage could occur. The structures used consist of equipped with supports hanging on rails, one behind the other, along the whole length of the spinning frame; they are equipped with a braking device, which prevents the bobbin from rotating too quickly [1].

b) Drafting unit: The drafting unit can be equipped with different types of fiber control devices and precisely: three-cylinder fiber control device with double apron (Figure 2, right) for yarns with medium and medium-fine counts, four-cylinder fiber control device with double apron (Figure 2, left) for yarns with fine and very fine
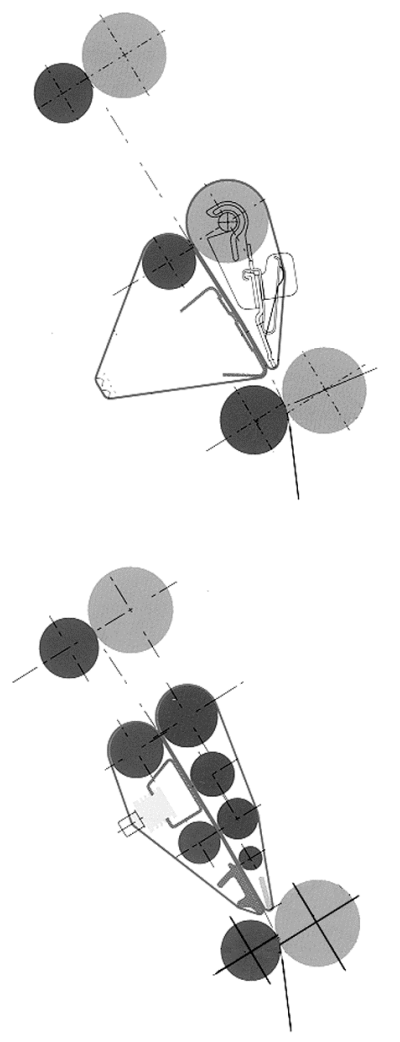

Figure 2. Drafting unit. 
counts with high evenness, resistance and elastic properties. The fiber mass entering the draft range is made of a slightly resistant fiber sliver since it contains only few fibers; for this reason the friction is reduced to a minimum. Two rotating aprons grant a suitable control; the upper apron compresses the material against the lower apron.

\section{The yarn tension in ring spinning machine [2]:}

In ring spinning as shown in Figure 3, tension develops in the yarn mainly because, to move the Traveler and the balloon length, $L_{b}$, around the common axis, and to wind the yarn onto the spinning bobbin, work must be done against the frictional force of the ring on the Traveler and of the Traveler on the yarn, as well as against the air drag on the Traveler and on the balloon length. This work is additional to that needed to overcome the friction of the spindle bearings and the air drag on the forming yarn package. The tensions in the yarn during ring spinning may be considered with respect to three zones: the winding zone, the balloon zone, and the yarn formation zone. The winding zone is the area in which the yarn length from Traveler to forming package develops a winding tension, $T_{W}$. In the balloon zone, tension occurs in the yarn length between the Traveler and lappet guide (often referred to as the balloon tension). This tension, at a given point on the balloon length, varies with amplitude (i.e., the radius of the point) measured from the common axis. At the ring and Traveler, the balloon tension is provided by $T_{R}$ and is related to $T_{W}$. In the yarn formation zone (i.e., the zone between the pigtail lappet guide and the front rollers of the drafting system), the yarn tension is termed the spinning tension, $T_{S}$, and is related to the balloon tension at the lappet guide, $T_{0}$ may be rewritten as:

$$
\begin{aligned}
& T_{0}=T_{S} \cdot \mathrm{e}^{K \cdot \theta} \\
& T_{W}=T_{R} \cdot \mathrm{e}^{\vartheta \cdot \alpha}
\end{aligned}
$$

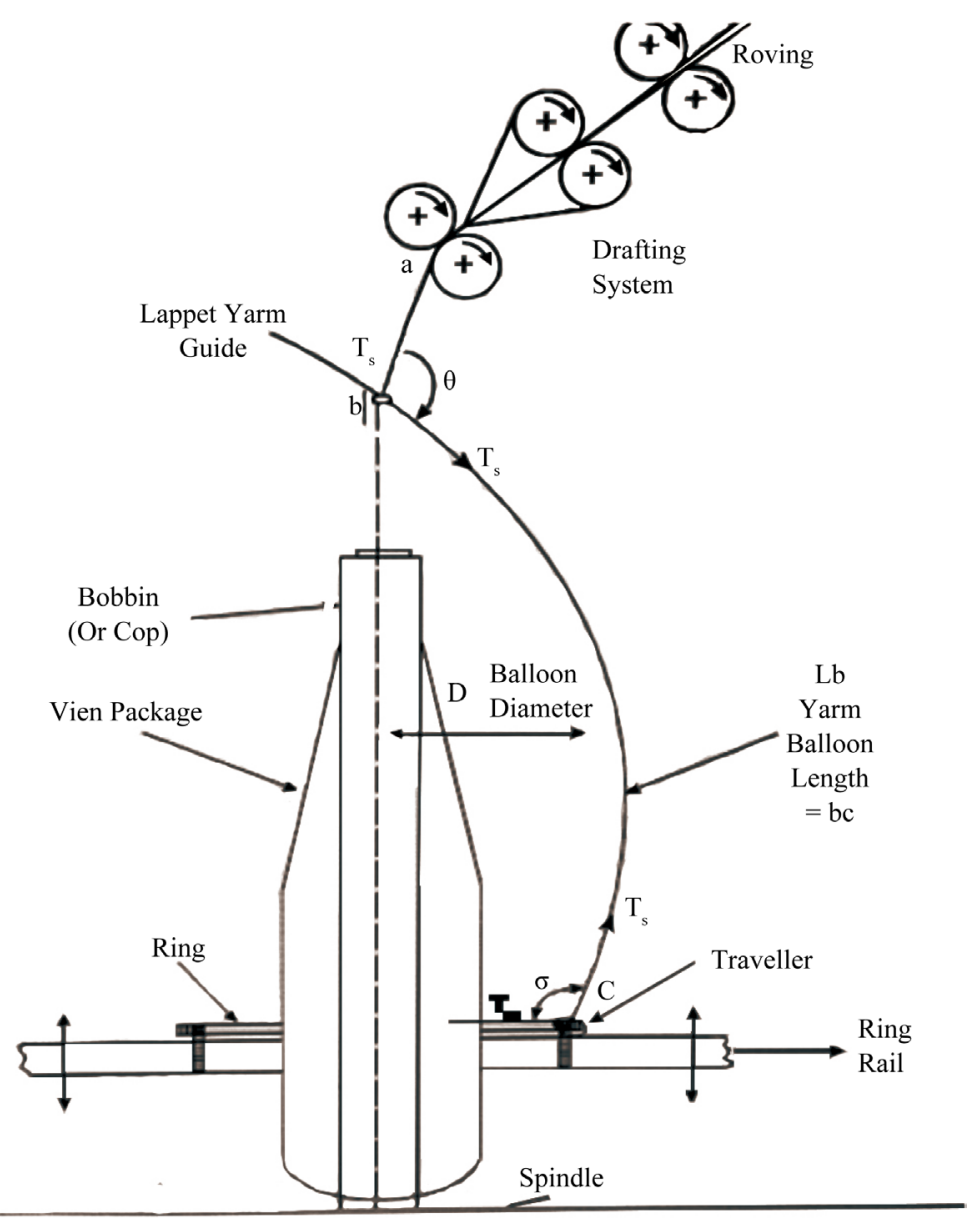

Figure 3. The yarn tension forces in ring spinning machine. 
To understand the physical causes of these tensions in the yarn, we need to consider the forces acting on the yarn in the three zones:

1) Yarn formation zone: the zone between the pigtail lappet guide and the front rollers of the drafting system.

2) Winding zone: the zone between the Traveler and the bobbin forming zone.

3) Balloon forming zone: the zone between the Traveler and lappet guide.

\section{Yarn formation zone:}

Although the yarn rotates around the inner circumference of the lappet guide at almost the same speed as the Traveler, the radius of the lappet guide is sufficiently small for any central forces generated to be ignored. The motion of the yarn between the lappet and front drafting rollers is therefore principally related to the velocity along its length (i.e., the thread line velocity). Consequently, the forces of interest are the air drag along its length, the tension at the lappet guide, and the resistance to bending around the guide. The air drag is proportional to the square of the thread line velocity, but this velocity is usually small as compared with the rotational velocity of the yarn. Thus, the force caused by the air drag along the yarn length is assumed to be negligible. The bending resistance due to the flexural rigidity of the yarn is many times smaller than $T_{0}$ and can also be omitted from further consideration $T_{0}$ is therefore the only effective force governing $T_{S}$ and, as a result, analysis of the forces present in ring spinning is usually concerned with the remaining two zones [2].

In the Figure 4:

Projection on the t-axis, we find:

$$
(T+\mathrm{d} T) \cdot \cos \mathrm{d} \theta-T-F_{f} \cdot \mathrm{d} S=0
$$

Projection on the n-axis, we find:

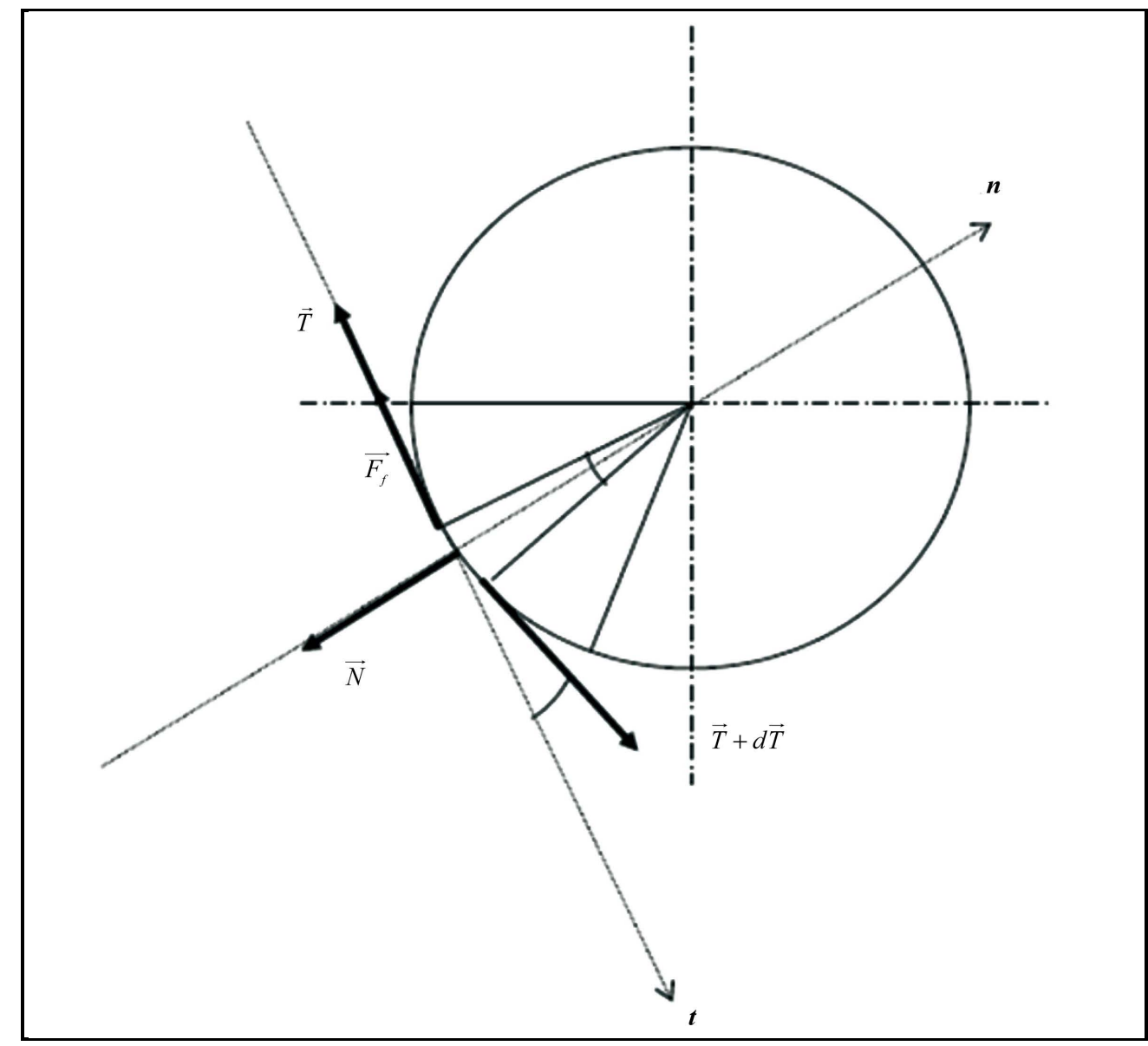

Figure 4. The yarn tension forces in yarn formation zone. 


$$
\begin{aligned}
& (T+\mathrm{d} T) \cdot \operatorname{sind} \theta-N \cdot \mathrm{d} S=0 \\
& \lim _{\varphi \rightarrow 0} \operatorname{sind} \varphi=0 \\
& \lim _{\varphi \rightarrow 0} \cos \mathrm{d} \varphi=1 \\
& \Rightarrow(T+\mathrm{d} T)-T=F_{f} \cdot \mathrm{d} S \\
& \Rightarrow F_{f}=\frac{\mathrm{d} T}{\mathrm{~d} S} \text { but } F_{f}=K \cdot N \\
& \Rightarrow K \cdot N=\frac{\mathrm{d} T}{\mathrm{~d} S} \\
& \Rightarrow N=T \cdot \frac{\mathrm{d} \theta}{\mathrm{d} S} \\
& \Rightarrow K \cdot T \cdot \mathrm{d} \theta=\mathrm{d} T \\
& \Rightarrow \int_{T_{S}}^{T_{0}} \frac{\mathrm{d} T}{T}=\int_{0}^{\theta} K \cdot \mathrm{d} \varphi \\
& \Rightarrow \frac{T_{0}}{T_{S}}=\mathrm{e}^{K \cdot \theta}
\end{aligned}
$$

\section{Winding zone:}

In steady running conditions, the Traveler presses against the bottom of the internal flange of the ring, as illustrated in Figure 5. The forces acting on the Traveler at the point of contact with the ring are also depicted. Strictly,
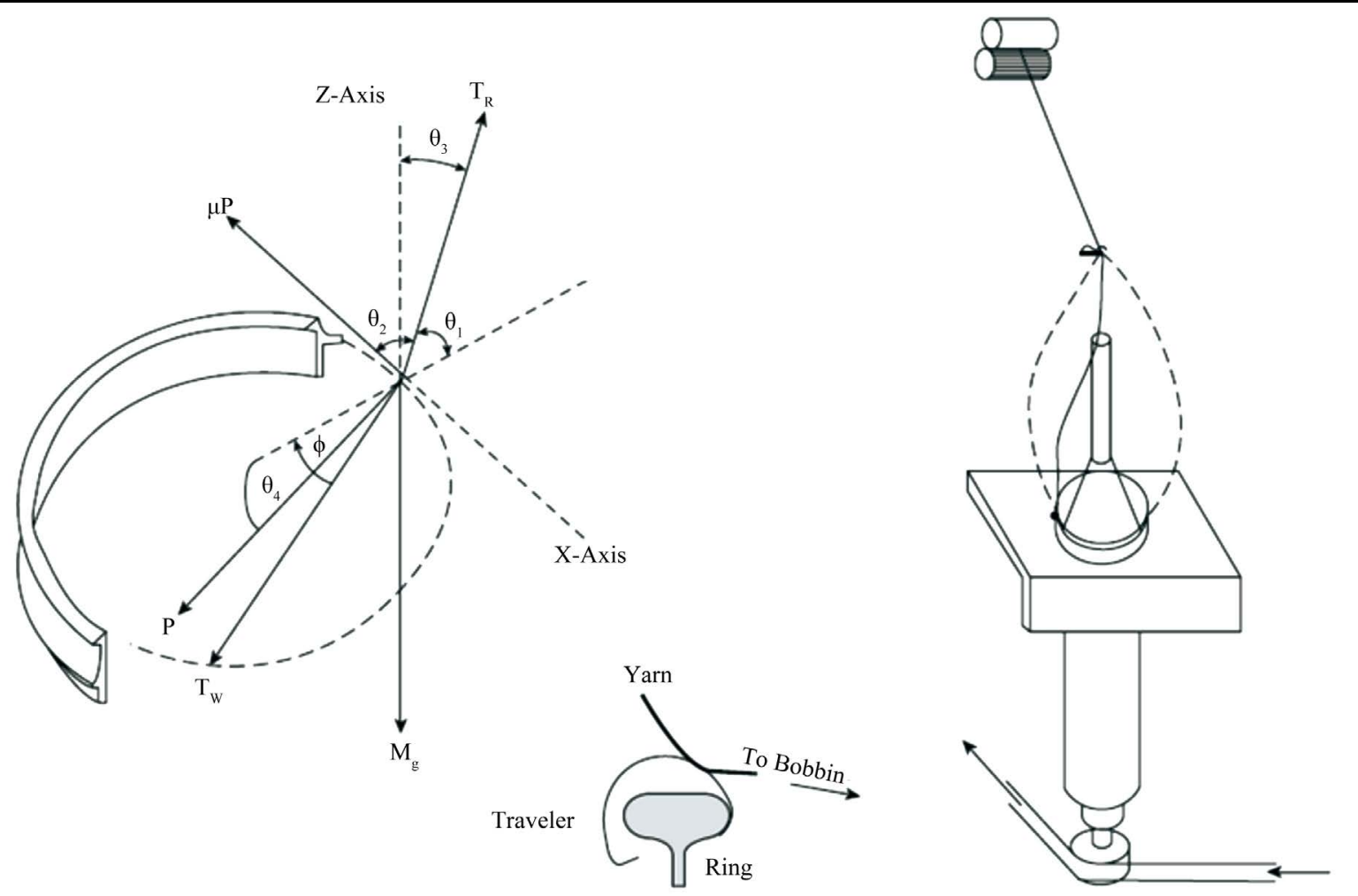

Figure 5. Forces acting on Traveler during steady running conditions. 
$T_{W}$ is not the true winding tension. This is because centripetal and air-drag forces act on the mass of the yarn length from the Traveler to the ring bobbin. It can be reasoned that the latter two effects negate each other and therefore can be neglected. The effect of the centripetal force is to change the path of the yarn from that of a tangent from the package to the Traveler, to one of a curve. The change, however, is small and, for the sake of simplicity, this centripetal force is also neglected [3].

Let us first consider tensions $T_{R}$ and $T_{W}$ and the other forces acting on the Traveler during steady running conditions (i.e., dynamic equilibrium). Resolving the forces shown in Figure 5 into their vertical and horizontal components gives [3]:

In the Figure 5:

Projection on the $y$-axis, we find:

$$
\begin{aligned}
& C+T_{R} \cdot \cos \theta_{1}-P \cdot \cos \theta_{4}-T_{W} \cdot \cos \varphi=0 \\
& \Rightarrow C=P \cdot \cos \theta_{4}+T_{W} \cdot \cos \varphi-T_{R} \cdot \cos \theta_{1}
\end{aligned}
$$

Projection on the $x$-axis, we find:

$$
\begin{aligned}
& T_{W} \cdot \sin \varphi-\mu \cdot P-T_{R} \cdot \cos \theta_{2}=0 \\
& \Rightarrow T_{W} \cdot \sin \varphi=\mu \cdot P+T_{R} \cdot \cos \theta_{2}
\end{aligned}
$$

Projection on the $z$-axis, we find:

$$
T_{R} \cdot \cos \theta_{3}=P \cdot \sin \theta_{4}+M \times g
$$

where:

$\Phi, \cdots, \theta_{1}$ : inclination angles powers for axes waypoint.

$C$ : centripetal force needed to keep the Traveler circulating around the ring and given the following relationship:

$$
C=M \cdot R \cdot \omega^{2}
$$

$P=$ reaction force of the ring and Traveler.

$R=$ ring radius.

$M g=$ weight of the Traveler.

$\mu=$ friction coefficient between ring and Traveler.

From Equations (7)-(9):

$$
\begin{aligned}
& T_{W}=T_{R} \cdot \mathrm{e}^{\vartheta \cdot \alpha} \Rightarrow T_{R}=T_{W} \cdot \mathrm{e}^{-\vartheta \cdot \alpha} \\
& \aleph=\mathrm{e}^{-\vartheta \cdot \alpha} \\
& \Rightarrow T_{R}=\aleph \cdot T_{W} \\
& \Rightarrow T_{R}=\frac{\aleph \times \mu \times C}{\sin \varphi \cdot \cos \theta_{4}+\mu \cdot \cos \varphi-\aleph \cdot\left(\mu \cdot \cos \theta_{1}+\cos \theta_{1} \cdot \cos \theta_{2}\right)} \\
& \sin \theta_{4}=\frac{\aleph \cdot \mu \cdot\left(T_{R} \cdot \cos \theta_{3}-M \cdot g\right)}{T_{W} \cdot\left(\sin \varphi-\aleph \cdot \cos \theta_{2}\right)}
\end{aligned}
$$

To simply the above equations and obtain an estimate for $T_{R}$, we can assume that the weight of the Traveler, $M g$, is negligible in comparison to $T_{R}$ so that $M g$ can be removed from the equations. From practical observation, it is also reasonable to assume that $\theta_{3}$ is such that $\cos \theta_{3}=1$. In the absence of air-drag,

$$
\begin{aligned}
& \theta_{2}=\frac{\pi}{2} \Rightarrow \cos \theta_{2} \rightarrow 0 \\
& \theta_{2} \rightarrow \frac{\pi}{2} \Rightarrow \cos \theta_{1} \rightarrow 0
\end{aligned}
$$




$$
\begin{aligned}
& T_{R}=\frac{\aleph \times \mu \times C}{\sin \varphi \cdot \cos \theta_{4}+\mu \cdot \cos \varphi} \\
& \sin \theta_{4}=\frac{\aleph \cdot \mu}{\sin \varphi} \\
& \cos \theta_{4}=\frac{\sqrt{\sin ^{2} \theta-\aleph^{2} \cdot \mu^{2}}}{\sin \varphi} \\
& \Rightarrow T_{R}=\frac{x \cdot \mu \cdot C}{\sqrt{\sin ^{2} \theta-\aleph^{2} \cdot \mu^{2}}+\mu \cdot \cos \varphi} \\
& \Rightarrow T_{w}=\frac{\mu \cdot C}{\sqrt{\sin ^{2} \theta-\aleph^{2} \cdot \mu^{2}}+\mu \cdot \cos \varphi}
\end{aligned}
$$

- It can be seen from Equations (9) and (10) that, when $\mu$ is small, such that $\sin \theta_{4} \rightarrow 0$ and $\cos \theta_{4} \rightarrow 1$, then $T_{R} \rightarrow \frac{\aleph \cdot \mu \cdot C}{\sin \varphi} \Rightarrow T_{W}=\frac{\mu \cdot C}{\sin \varphi}$.

We can see, too, that frictional drag of the ring on the Traveler is dependent on $\varphi, \mu p=T_{W} \sin \varphi$ In general, then, as the package builds up, the winding tension will decrease with the lead angle $\varphi$ determined by the frictional drag of the ring on the Traveler, and also of the Traveler on the yarn, as the latter passes through the former to the package. During a typical bobbin build, $\sin \varphi$ can vary from between 0.45 and 0.5 (empty) to between 0.9 and 0.95 (full), so $T_{W}$ can almost halve in size during the package build. The variation of $T_{W}$ during the package build will therefore give a package hardness, decreasing in hardness from the inside to outer layers. With a given ring diameter, this governs the amount of yarn that can be wound onto a bobbin. For a fixed spindle speed and ring diameter, altering the Traveler friction and mass will provide a particular winding tension, say, for a package of required hardness. For example, if $\mu$ is small, a heavier Traveler will be needed than when $\mu$ is large. If $\mu$ is fixed, the tension becomes governed by the Traveler mass. Thus, the selection of the Traveler will depend on the maximum tension that the yarn will withstand when winding on an empty bobbin [3].

Figure 6 shows two possible running positions for the Traveler. The normal running position is of the Traveler contacting the inside of the ring. However, if too light a Traveler is used, $T_{w} \cos \theta_{4}$ becomes greater than the centripetal force, $C$, and the Traveler runs on the outside of the ring; this is termed outside tracking. With regular running, $\theta_{4}<90^{\circ}$ and $\cos \theta_{4}$ is positive. With outside tracking, $\theta_{4}>90^{\circ}$ and $\cos \theta_{4}$ is negative. However, $T_{r}$ and $T_{w}$ would still be positive. High tension fluctuations occur, making the situation unstable.

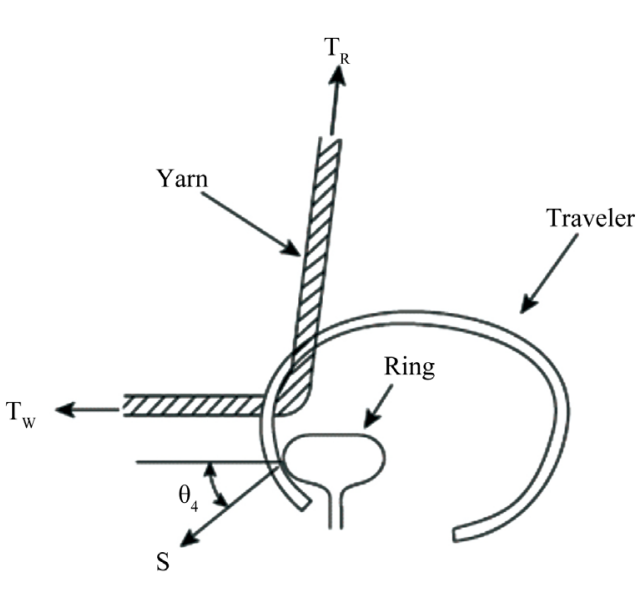

(a) $\theta_{4}<90^{\circ}$

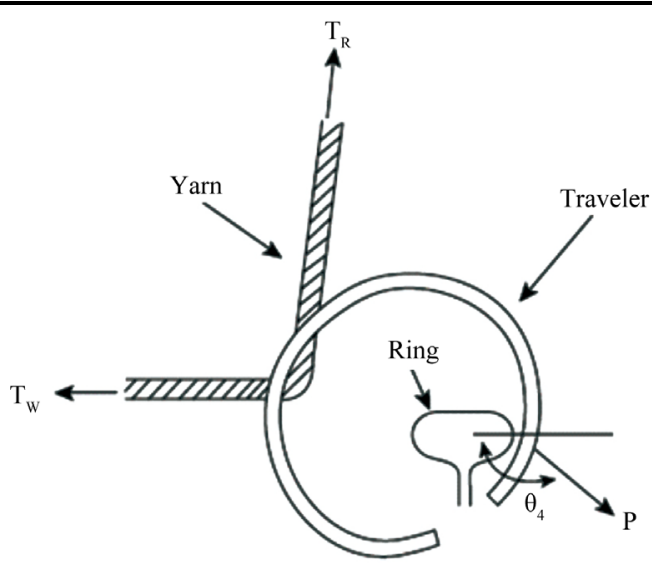

(b) $\theta_{4}<90^{\circ}$

Figure 6. Normal running and outside tracking of Traveler. 


\section{Balloon forming region (Balloon forming zone):}

The Figure 7 shows next three dimensional areas where the balloon is positioned formed when a ring spinning process, so that the point 0 represents the first nodal point of the balloon and that form the top and in order to study the dynamics of Affecting the balloon on a string, we first choose the appropriate phrase and the coordinates are: $X, Y, Z$.

And which rotates with the balloon's rotation around the $Z$, which is the main focus in this sentence and is applicable on the axis and the axis spindle thread prompt axis, while the $X$-axis is parallel to the radius of the ring.

Then we work on the choice of elemental part of the balloon string length dl so that symbolizes the distance from the rotation axis $Z$ symbol $r$, therefore, be the forces affecting it are:

Centrifugal force (The centripetal force);

Carioles force (The carioles force);

So that the movement of the preceding two thread balloon;

Thoughtful element weight (Weight of studying unit);

Air force effect (Air drag force) adverse movement thread;

Bending resistance shown by the body resulting from the thread hardness of raw materials consisting of the output string.

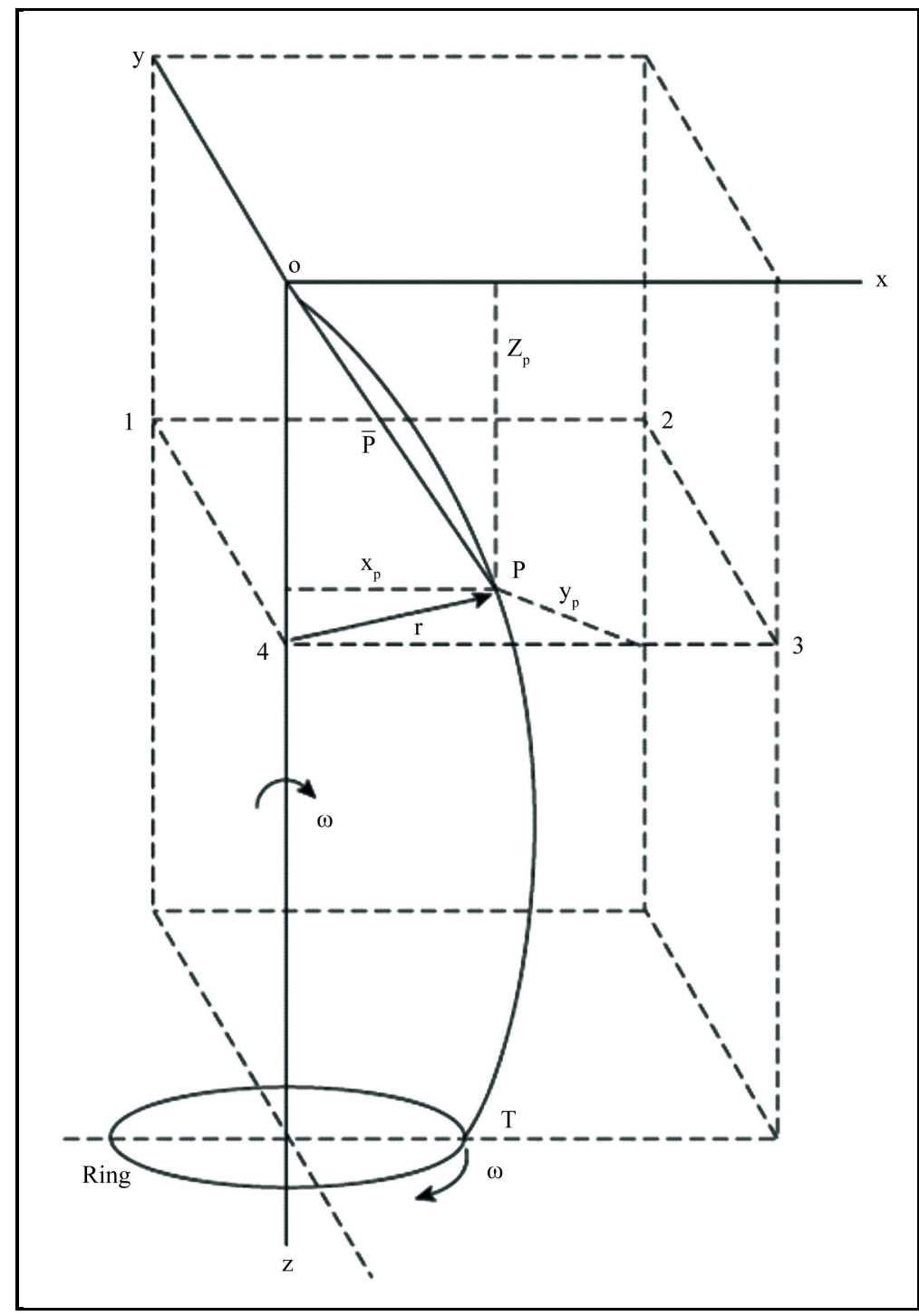

Figure 7. Balloon forming zone. 
So that will be discussed each force separately.

Central to the power of expulsion two vehicles [2]-[11]:

Vehicle first: affect the racial body so that it works to make a curvature toward the outside, so that the value of the vehicle based on the linear speed body balloon and bound to the downside, resulting from the direction of the element of the string prompt and even Traveler, so that the speed of a speed-cylinder supply a relatively small amount when compared spindle rotational speed, so it can neglect the power of this vehicle.

The second vehicle: affect the horizontal component and the vehicle body is working to push the thread outward away from the main axis, and because of the high-speed rotational spindle and Traveler this vehicle are taken into account during the dynamic study.

As for the carioles force it is the power emerging as a result of the presence of the relative movement between Traveler and cylinder supply (because of the speed difference linear high), and as a result, the body of the thread will move helical movement starting from half a smaller diameter and radius which followed as a result of the impact of three vehicles to speed areas shown in Figure 8:

- Radial velocity $V_{y}$.

- Speed perimeter balloon $V_{t r}$.

- Vertical speed, which is heading towards Traveler and episode $V_{f}$.

When the movement of racial part of the thread of the balloon ranging from small to half the maximum diameter radius, will lead to the creation of an increase in the value of the kinetic energy EK (Kinetic energy), which is given according to the following relationship:

$$
\mathrm{EK}=\frac{1}{2} \cdot m \cdot d l \cdot r^{2} \cdot \omega^{2}
$$

where:

$r$ : polar component thoughtful about the vertical axis dimension;

$\omega$ : rotational speed of the balloon and string are the same rotational speed of the rings;

$m$ : mass of thread balloon.

Affecting the deliberate element of force, which cause increased kinetic energy value is the vehicle Carioles force associated with the speed of the circular balloon, and when you increase the value of the kinetic force, the Department of force is toward direct rotation so increases the energy value, and starting from the thread prompt and even the maximum radius of the balloon, but when the movement of the maximum radius and even Traveler the vehicle Carioles force will be opposite to the direction of rotation element studied thus lower the value of the kinetic energy (and therefore when it is half the maximum diameter of the balloon is greater than the radius of the loop) [3].

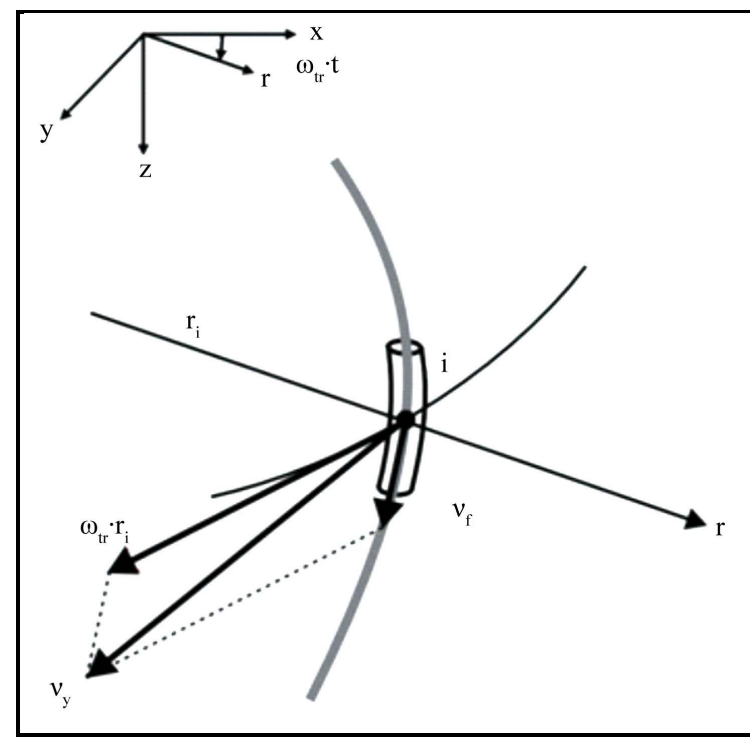

Figure 8. Forces in the balloon zone. 
The second vehicle of the force they affect the internal radial studied element and is about the speed of the transmission component of the vector and even Traveler.

Vehicle radial force resulting from radial velocity given to the following relationship:

$$
F_{r}=2 \cdot m \cdot V_{t} \cdot \omega
$$

The tangential force resulting from the tangential speed of the balloon body are given the following relationship:

$$
F_{t}=2 \cdot m \cdot V_{t r} \cdot \omega
$$

Since it is in the ring spinning process are rotational speed is much larger than the rotational speed of the cylinder supply clouds device spindle, the Carioles force vehicles can be neglected.

And similarly, the effect of the air force for two vehicles are:

acting on a body thread balloon force;

air resistance to rotation studied element.

The second vehicle is proportional to the rotational speed square and are of great importance in our study, the fact that the reluctance body balloon air to effect gain additional robustness about undergoing stress tensile, as and consider the effect of the air is a constant value over the entire length of the thread of the balloon and that as (The thread is a regular cylindrical body section and constant diameter over the entire length) can thus calculate the approximate value of the effect of the air force of law through the following:

$$
\mathrm{AD}=\frac{1}{2} \cdot \rho_{A} \cdot \varepsilon \cdot d \cdot V_{N}^{2}
$$

where:

$\rho_{A}$ : the density of the air surrounding the body of the balloon;

$\varepsilon:$ air impact factor;

$d$ : diameter thread;

$V_{N}$ : natural speed of the element studied and are the same speed transmission component of the vector and even Traveler.

The effect of the air coefficient $\varepsilon$ continued to speed and relative to the diameter of the thread and dynamic air, so through discretionary operations during the research process to force air impact can be seen that the value of $\varepsilon$ during the technological process.

In practice, the effect of the air is not uniform over the entire length of the thread of the balloon and the reason for this is due to the existence of the phenomenon (hairiness) so that the parties to the side of a breakthrough in the ocean air space thread balloon capillaries work thus differing air spaces effect on the body Floss.

As for the weight element lessons can neglect the fact that it is a very small value, and as the full flexibility element, thus can be neglected both the weight and strength of the crease.

Study the tensile forces acting on the thread of the balloon in this region will depend on two pillars:

1) Tightening the balloon in the absence of air effect (case full washroom).

2) Tightening the balloon, taking into account the effect of the air.

1. (Balloon tension in the absence of air drag) [2]-[11]:

Note from the previous paragraph that tightening the balloon is a variable value and because of the variety after thoughtful element axis vertical $Z$ during the transition from the thread prompt and even Traveler, and for pulling the balloon relationship at any point of it, we are taking a length element $\mathrm{d} l$ as shown in Figure 9 and is located After the $x$ from the vertical axis.

The Figure 10 shows that the curvature of the length of the balloon is in the plane $X-Z$ and the so-called pivot level (Axial plane).

Assuming that $m$ is the mass of a single length of thread, therefore be active forces acting on this item:

- the second vehicle to centrifugal force;

- tensile strength at both ends of the element and take symbols.

The following: $(T),(T+d T)$.

Projection on the $Z$-axis, we find:

In the Figure 9: 


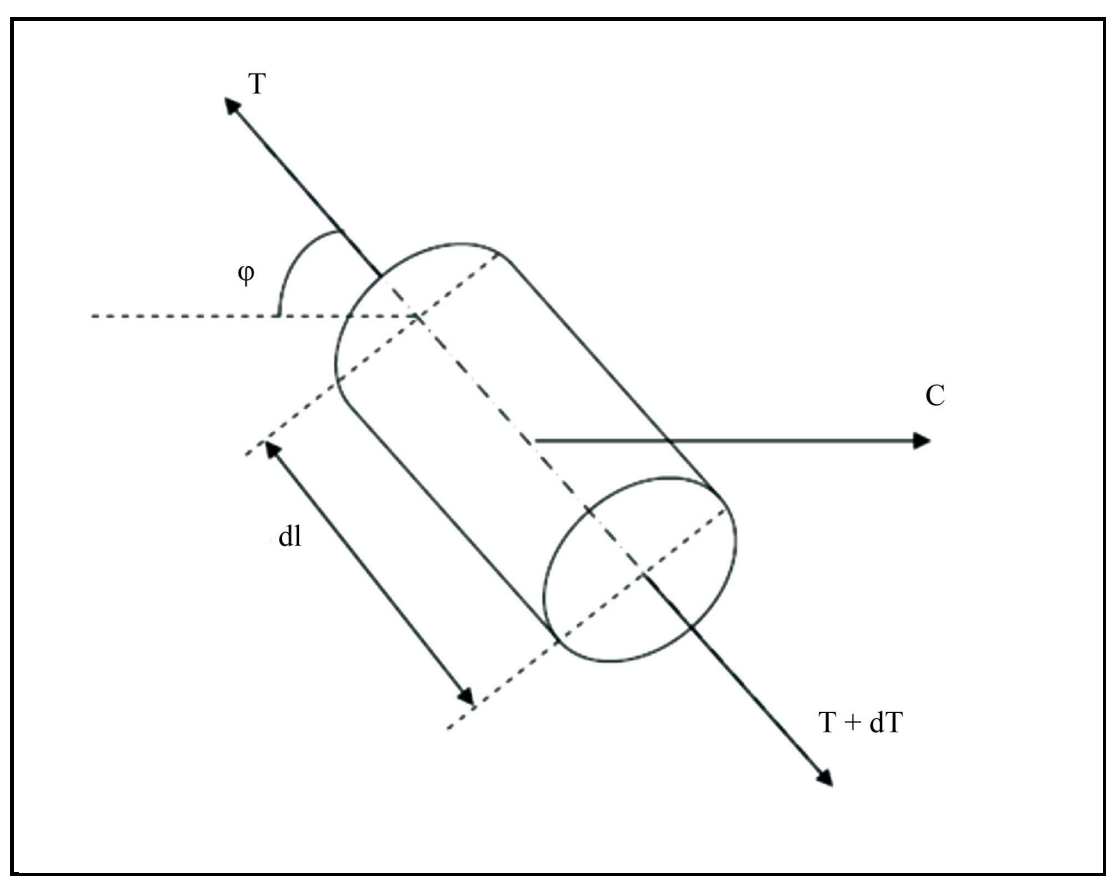

Figure 9. Length element $\mathrm{dl}$ and is located after the $x$ from the vertical axis.

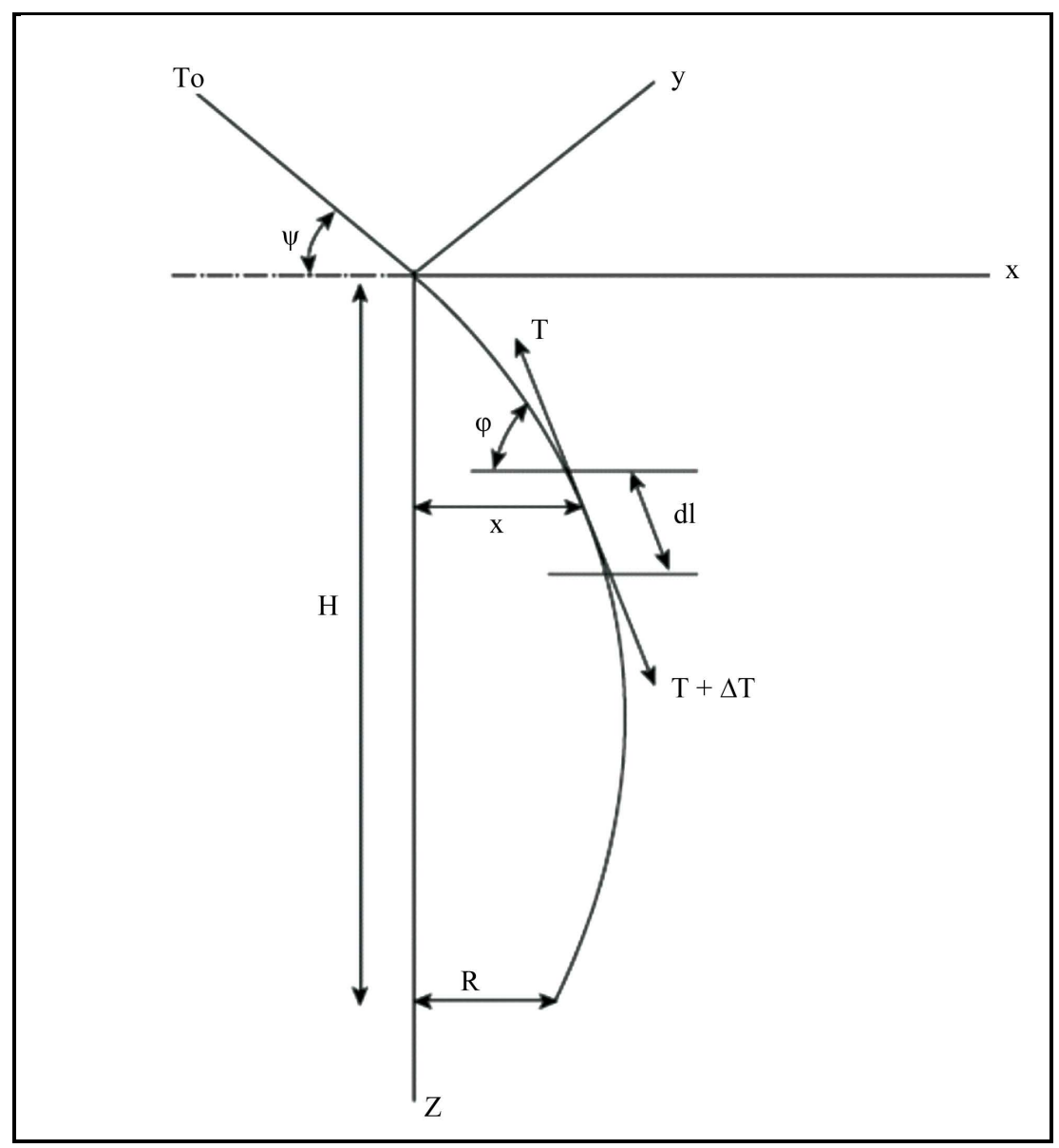

Figure 10. Balloon tension forces. 


$$
\begin{aligned}
& T \cdot \sin \varphi+\mathrm{d}(T \cdot \sin \varphi)-T \cdot \sin \varphi=0 \\
& \Rightarrow \mathrm{d}(T \cdot \sin \varphi)=0 \\
& C+[T \cdot \cos \varphi+\mathrm{d}(T \cdot \cos \varphi)]-T \cdot \cos \varphi=0 \\
& \Rightarrow C=T \cdot \cos \varphi-[T \cdot \cos \varphi+\mathrm{d}(T \cdot \cos \varphi)] \\
& \Rightarrow \mathrm{d}(T \cdot \cos \varphi)=-m \cdot \mathrm{d} l \cdot x \cdot \omega^{2} \\
& \cos \varphi=\frac{\mathrm{d} x}{\mathrm{~d} l} \\
& \frac{\mathrm{d}}{\mathrm{d} l}=\frac{\mathrm{d} \varphi}{\mathrm{d} l} \cdot \frac{\mathrm{d}}{\mathrm{d} \varphi} \\
& T \cdot d(\sin \varphi)+\sin \varphi \cdot \frac{\mathrm{d} T}{\mathrm{~d} \varphi}=0 \\
& \Rightarrow T \cdot \cos \varphi+\sin \varphi \cdot \frac{\mathrm{d} T}{\mathrm{~d} \varphi}=0 \\
& \Rightarrow \frac{\mathrm{d} \varphi}{\tan \varphi}=-\frac{\mathrm{d} T}{T} \\
& \Rightarrow \cos \varphi \cdot \frac{\mathrm{d} T}{\mathrm{~d} l}-T \cdot \sin \varphi \cdot \frac{\mathrm{d} \varphi}{\mathrm{d} l}=-m \cdot x \cdot \omega^{2} \\
& \Rightarrow T \cdot \sin \varphi \cdot \mathrm{d} \varphi-\cos \varphi \cdot \mathrm{d} T=m \cdot \mathrm{d} l \cdot x \cdot \omega^{2} \\
& \Rightarrow-\frac{\mathrm{d} T}{\cos \varphi}=m \cdot \mathrm{d} l \cdot x \cdot \omega^{2} \\
& \Rightarrow-\mathrm{d} T=m \cdot x \cdot \omega^{2} \cdot \mathrm{d} x \\
& \int_{T}^{T_{0}} \mathrm{~d} T=\int_{0}^{x}\left(-m \cdot x \cdot \omega^{2}\right) \mathrm{d} x \\
& \Rightarrow T_{0}-T=-m \cdot \omega^{2} \cdot \int_{0}^{x} x \cdot \mathrm{d} x \\
& \Rightarrow-T_{0}+T=-m \cdot \omega^{2} \cdot\left[\frac{1}{2} \times x^{2}\right] \\
& \Rightarrow T=T_{0}-\frac{1}{2} \cdot m \cdot x^{2} \cdot \omega^{2} \\
& \int_{\varphi_{0}}^{\varphi} \frac{\mathrm{d} \varphi}{\tan \varphi}=\int_{T_{0}}^{T}-\frac{\mathrm{d} T}{T} \\
& \Rightarrow \int_{\varphi_{0}}^{\varphi} \frac{\mathrm{d} \varphi}{\frac{\sin \varphi}{\cos \varphi}}=-\int_{T_{0}}^{T} \frac{\mathrm{d} T}{T} \\
& \Rightarrow \int_{\varphi_{0}}^{\varphi} \frac{\cos \varphi}{\sin \varphi} \mathrm{d} \varphi=-\int_{T_{0}}^{T} \frac{\mathrm{d} T}{T}
\end{aligned}
$$




$$
\begin{aligned}
& \Rightarrow \log [\sin \varphi]_{\varphi_{0}}^{\varphi}=-\log [T]_{T_{0}}^{T} \\
& \Rightarrow \log \frac{\sin \varphi}{\sin \varphi_{0}}=\log \frac{T_{0}}{T} \\
& \Rightarrow \frac{\sin \varphi}{\sin \varphi_{0}}=\frac{T_{0}}{T}
\end{aligned}
$$

where:

$\varphi_{0}$ : inclination angle of the thread element on the horizon prompt the bottom of the thread.

Previous relations show that the tensile strength be valuable down the maximal string prompt and they are of value at the maximum radius of the balloon.

And from a technological standpoint, the tensile value bottom thread prompt with serious damage to the thread, and that it works to bring reluctant to spread even cylinders drag the front of the drag system, and this leads to a change in yarn output specifications and increase the value of the different twisting coefficient.

And when we have [2]-[11]:

$$
\begin{aligned}
& \Rightarrow T=T_{R} \\
& \Rightarrow T_{R}=T_{0}-\frac{1}{2} \cdot m \cdot R^{2} \cdot \omega^{2} \\
& \Rightarrow T_{0}=T_{R}+\frac{1}{2} \cdot m \cdot R^{2} \cdot \omega^{2} \\
& T_{R}=\frac{\mu \cdot C}{\sqrt{\sin ^{2} \theta-\aleph^{2} \cdot \mu^{2}}+\mu \cdot \cos \varphi} \\
& \Rightarrow T_{0}=\frac{\mu \cdot C}{\sqrt{\sin ^{2} \theta-\aleph^{2} \cdot \mu^{2}}+\mu \cdot \cos \varphi}+\frac{1}{2} \cdot m \cdot R^{2} \cdot \omega^{2}
\end{aligned}
$$

- And note from this equation that the tensile value $T_{0}$ depends on:

1) angle tweaking;

2) friction between Traveler and loop coefficient;

3) the angular velocity of the rings;

4) floss operator type.

\section{Air tightening effect on the floss (the effect of air drag on yarn tension) [3]:}

As we have seen previously, and the absence of the effect of the air on the balloon, the length of the balloon is located in the plane $X$ - $Z$ plane and the vertical is the pivot level, but in case the effect of air balloon on a string, the latter will be inclined from the vertical plane through the thread thoughtful element move oriented to Traveler and pipe, so you must study the length of the balloon within the three-dimensional space as shown in Figure nearby so that it has been replaced by $r$ instead of $x$ in the fact that the level of relations does not balloon is positioned perpendicular to the $X$ axis.

- For careful analysis of the impact of the air forces pulling the thread, you must work to identify the angles $\theta_{1}$ through $\theta_{4}$ corner through a loop and the effect of forces on the Traveler.

- And also that the angle $\varphi$ a lapping process, so note the presence of great difficulty from an engineering standpoint in the study of the effect of the air forces pulling the thread.

- And when the air effect on the length of the balloon, it generates a horizontal vehicle to flatten the form with the outcome of the tensile strength resulting from the former, vehicle to flatten down and causing an increase in the tensile force generated within the thread.

- Assuming that $D$ is the power of air resistance added, the relationship (10) becomes as follows:

$$
T_{R}=\frac{\aleph \times\left(\mu \cdot C+D \cdot \cos \theta_{4}\right)}{\sin \varphi \cdot \cos \theta_{4}+\mu \cdot \cos \varphi-\aleph \cdot\left(\mu \cdot \cos \theta_{1}+\cos \theta_{1} \cdot \cos \theta_{2}\right)}
$$




$$
\begin{gathered}
\mathrm{AD}=\frac{1}{2} \cdot \rho_{A} \cdot \varepsilon \cdot d \cdot V_{f}^{2} \\
V_{N}=r \cdot \omega \cdot \cos \alpha
\end{gathered}
$$

where $\alpha_{1}$ is the angle of inclination angle beam speed VN holder for the $y$-axis, thus given mechanical energy arising as a result of the impact of the air within the thread following relationship:

$$
\begin{gathered}
\mathrm{MK}=\frac{1}{2} \cdot \rho_{A} \cdot \varepsilon \cdot d \cdot V_{N}^{2} \cdot \omega \cdot r \\
\Rightarrow \mathrm{MK}=\frac{1}{2} \cdot \rho_{A} \cdot d \cdot \varepsilon \cdot \mathrm{d} l \cdot \omega^{3} \cdot r^{3} \cdot \cos ^{2} \alpha_{1} \\
\mathrm{MK}=\int_{0}^{L_{b}} \frac{1}{2} \cdot \rho_{A} \cdot d \cdot \varepsilon \cdot \mathrm{d} l \cdot \omega^{3} \cdot r^{3} \cdot \cos ^{2} \alpha_{1} \\
\Rightarrow \mathrm{MK}=\frac{1}{2} \cdot \rho_{A} \cdot d \cdot \varepsilon \cdot \omega^{3} \cdot \int_{0}^{L_{b}} r^{3} \cdot \cos ^{2} \alpha_{1} \cdot \mathrm{d} l
\end{gathered}
$$

And when the subject of the previous episode, the relationship is equal opposite force is the power of the air effect on Traveler, and as Traveler rotational speed equal to the rotational speed of the thread of the balloon, it can be written the following relationship:

Mechanical energy generated by the impact of the air on the thread = mechanical energy generated by the impact on air Traveler

$$
\begin{gathered}
\Rightarrow T_{R A} \cdot \cos \theta_{2} \cdot R \cdot \omega=\frac{1}{2} \cdot \rho_{A} \cdot d \cdot \varepsilon \cdot \omega^{3} \cdot \int_{0}^{L_{b}} r^{3} \cdot \cos ^{2} \alpha_{1} \cdot \mathrm{d} l \\
\Rightarrow T_{R A} \cdot \cos \theta_{2}=\frac{\rho_{A} \cdot d \cdot \varepsilon \cdot \omega^{3}}{2 \cdot R} \cdot \int_{0}^{L_{b}}\left[r^{3} \cdot \cos ^{2} \alpha_{1}\right] \cdot \mathrm{d} l
\end{gathered}
$$

We note that all the parameters depend on the shape of the balloon and the tensile strength and the TRA in order to facilitate the study can be considered that the shape of the balloon to keep away from the $Z$-axis is given in accordance with the sine equation:

$$
r=r_{\max } \cdot \sin \left(\frac{2 \cdot \pi \cdot z}{\lambda}\right)
$$

where:

$z$ : the vertical dimension of the element studied for prompt string.

$\lambda$ : wavelength and, as the effect of the air force affecting the horizontal level we have:

$$
H=\frac{\lambda}{2}, \quad \alpha_{1}=0
$$

And using the Pythagorean relationship racist lengths down the thread prompt directly, we find that:

$$
\mathrm{d} l^{2}=\mathrm{d} r^{2}+\mathrm{dz} z^{2}
$$

Thus becoming the relationship (20) as follows:

$$
\begin{gathered}
T_{R A} \cdot \cos \theta_{2}=\frac{\rho_{A} \cdot d \cdot \varepsilon \cdot \omega^{3}}{2 \cdot R} \cdot\left\{\int_{0}^{L_{b}} r_{\max }^{3} \cdot \sin ^{3}\left(\frac{2 \cdot \pi \cdot z}{\lambda}\right) \cdot 2 \cdot H \sqrt{\left[1+\frac{4 \cdot \pi \cdot r_{\max }^{2}}{\lambda^{2}} \cdot \cos ^{2}\left(\frac{2 \cdot \pi \cdot z}{\lambda}\right)\right]} \cdot \frac{\mathrm{d} l}{\mathrm{~d} z}\right\} \\
T_{R A} \cdot \cos \theta_{2}=\frac{\rho_{A} \cdot d \cdot \varepsilon \cdot \omega^{2} \cdot H \cdot r_{\max }^{3}}{4 \cdot R}=D
\end{gathered}
$$


And considering that $\theta_{1}=90^{\circ}$ and that $\theta_{3}=90^{\circ}-\theta_{2}$ we get the following relationship:

$$
\begin{gathered}
T_{R}=\cos \theta_{2}=\frac{\aleph \cdot\left(\mu \cdot C+D \cdot \cos \theta_{4}\right)}{\sin \varphi \cdot \cos \theta_{4}+\mu \cdot \cos \varphi}=D \\
\aleph=\mathrm{e}^{-\vartheta \cdot \alpha} \\
\Rightarrow T_{R}=\cos \theta_{2}=\frac{\left(\mu \cdot C+D \cdot \cos \theta_{4}\right)}{\left(\sin \varphi \cdot \cos \theta_{4}+\mu \cdot \cos \varphi\right) \cdot \mathrm{e}^{\vartheta \cdot \alpha}}=D
\end{gathered}
$$

Note from the previous relationship that tensile strength thread before suit reverse with:

The coefficient of friction between the ring and $\mu$.

The coefficient of friction between the body and the external thread metal thread prompt $\theta$.

So that friction made in the previous areas will greatly affect the superficial thread and specifically degree, so that conducted a very large number of research related to the most appropriate type of metal that achieves low coefficient of friction between the value of metallic thread and prompt thread formed with the body in order to achieve technological process and improve product quality.

\section{Experimental results and measurements:}

\section{The strength force testing:}

We took yarn with the annular Ne 12 to measure the tensile strength in the thread which is one of the most important tests of the mechanical properties of the filaments by the following:

\section{At the level of a single pipe [4]:} form:

Was taking a pipe and randomly from the annular spinning machine, so that has been tested in the following

- The removal of two layers of yarn from the outer surface of the pipe in order to get rid of the influence of external factors.

- has been taking a series of samples of 3 and a length of $50 \mathrm{~cm}$ after two laps of the outer surface of approximately $100 \mathrm{~m}$.

- After about $200 \mathrm{~m}$ from the previous samples, the test was performed on three other samples length of $50 \mathrm{cmas}$ shown in Table 1.

- After about $400 \mathrm{~m}$ from the previous sample, the test was performed on 3 samples length $50 \mathrm{~cm}$ Table 1 shows Strength force testing results on previous samples:

As shown in Figure 11 note of curves that pulling the thread changed significantly in relation to the diameter lapping (tensile increasingly smaller the diameter lapping).

- This is due to the low linear velocity of the ring, resulting in the decline in the number of twisting thus change in tensile strength thread.

Note from the graph devaluation of tensile strength of the pipe string from inside and around the outside, and the reason for this is due to the low linear speed of the rings. And the consequences of this decline in the number of low thus affected tensile strength.

\section{At the level of a set of pipes:}

This test has been in accordance with the following conditions as shown in Table 2:

- The initial sample length: $L V=50 \mathrm{~cm}$.

- The number of pipes taken for testing: $K=7$.

- The number of test samples from each pipe: $l=5$.

Thus the number of total samples $(n=5 \cdot 7=35)$ and is from the point of view of statistics and quality control enough to consider that the distribution of values behave normal distribution, which takes the form of a bell, which is subject to the experimental base, and when measuring the values of tensile strength for these samples, resulted we have the following Table 2:

$$
\begin{gathered}
\bar{X}_{i}=\frac{\sum_{i=1}^{n} X_{i}}{n} \\
S_{i}^{2}=\frac{\sum_{i=1}^{n}\left(X_{i}-\bar{X}_{i}\right)^{2}}{n-1}
\end{gathered}
$$


Table 1. Results of tensile strength thread (CN).

\begin{tabular}{cccc}
\hline SAMPLE & After about $100 \mathrm{~m}$ & After about $200 \mathrm{~m}$ & After about $400 \mathrm{~m}$ \\
\hline First test & $730[\mathrm{CN}]$ & $780[\mathrm{CN}]$ & $805[\mathrm{CN}]$ \\
Second test & $740[\mathrm{CN}]$ & $785[\mathrm{CN}]$ & $832[\mathrm{CN}]$ \\
Third test & $720[\mathrm{CN}]$ & $775[\mathrm{CN}]$ & $841[\mathrm{CN}]$ \\
average & $730[\mathrm{CN}]$ & $780[\mathrm{CN}]$ & $826[\mathrm{CN}]$ \\
\hline
\end{tabular}

Table 2. Results of tensile strength thread of the different pipes.

\begin{tabular}{|c|c|c|c|c|c|c|c|}
\hline Sample & 1 & 2 & 3 & 4 & 5 & $S_{i}^{2}$ & $\left(\bar{X}_{i}-\overline{\bar{X}}\right)^{2}$ \\
\hline 1 & 802 & 831 & 778 & 770 & 734 & 1315 & 190.44 \\
\hline 2 & 735 & 767 & 748 & 757 & 844 & 1840.7 & 707.56 \\
\hline 3 & 818 & 970 & 722 & 788 & 777 & 8714 & 331.24 \\
\hline 4 & 888 & 799 & 842 & 797 & 774 & 2048.5 & 538.24 \\
\hline 5 & 710 & 717 & 734 & 680 & 730 & 459.2 & 6822.76 \\
\hline 6 & 892 & 739 & 883 & 811 & 959 & 7086.2 & 3600 \\
\hline 7 & 826 & 842 & 776 & 849 & 799 & 913.3 & 466.56 \\
\hline & & & & & \multicolumn{2}{|c|}{22394.9} & 12656.8 \\
\hline
\end{tabular}

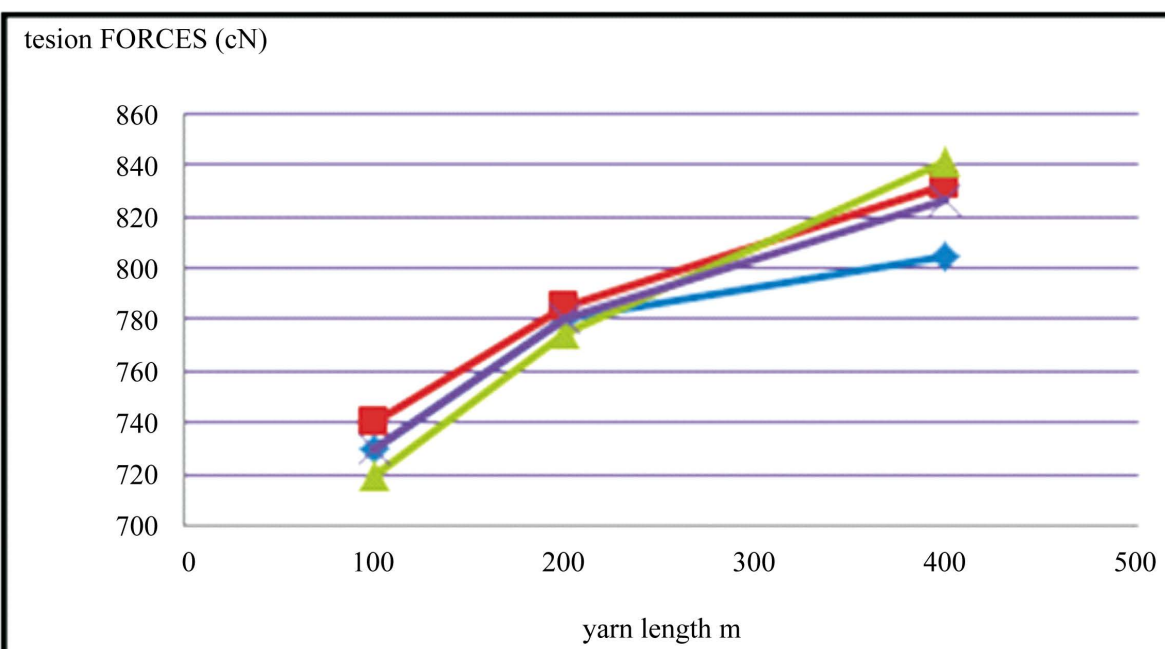

Figure 11. The strength force testing.

$$
\begin{aligned}
\overline{\bar{X}} & =\frac{\sum X_{i j}}{K \cdot l} \\
\overline{\bar{X}} & =\frac{802+831+\cdots+849+799}{7 \times 5}=796.8[\mathrm{gram}] \\
S_{b}^{2} & =\frac{\sum\left(\bar{X}_{i}-\overline{\bar{X}}\right)^{2}}{K-1}
\end{aligned}
$$




$$
\begin{aligned}
& \Rightarrow S_{b}^{2}=\frac{12656.8}{6}=10547.33 \mathrm{gram}^{2} \\
& S_{W}^{2}=\frac{\sum S_{i}^{2}}{K} \\
& \Rightarrow S_{W}^{2}=\frac{22394.9}{7}=3199.2743 \mathrm{gram}^{2} \\
& S_{e}^{2}=\frac{S_{b}^{2}-S_{W}^{2}}{l} \\
& \Rightarrow S_{e}^{2}=\frac{10547.33-3199.2743}{5}=1469.61114 \text { gram }^{2} \\
& \Rightarrow S_{e}=\sqrt{S_{e}^{2}}=\sqrt{1469.61114}=38.336 \text { gram }
\end{aligned}
$$

Homogeneity test [5]:

FB can homogeneity coefficient is calculated by the following law:

$$
F_{B}=\frac{S_{b}^{2}}{S_{W}^{2}} \Rightarrow F_{B}=\frac{10547.33}{1469.61114}=3.2968
$$

In order to ensure the statistical rate of $95 \%$ can extract the marginal value of $F$ tables so that the limit values for secondary $f_{1}$ and $f_{2}$ : can be calculated by the following laws:

$$
\begin{gathered}
f_{1}=K-1 \Rightarrow f_{1}=6 \\
f_{2}=K .(l-1) \Rightarrow f_{2}=7 \cdot(4)=28 \\
F=2.44 \Rightarrow F<F_{B}
\end{gathered}
$$

Thus article heterogeneous in terms of tensile strength.

Confidence-average:

To ensure the statistical rate of $95 \%$ and for the degree of freedom $f_{1}=6$ it can extract the parameter value $(t)$ of the table, thus we have [5]:

$$
t=2.45
$$

Since the material is homogeneous in terms of tensile strength, it can be applied to the following equation for the absolute amplitude of the field $q$ trust account:

$$
\begin{gathered}
q=t \cdot \sqrt{\frac{S_{b}^{2}}{K}+\frac{S_{W}^{2}}{K \cdot l}} \\
q q=2.45 \times \sqrt{\frac{10547.33}{7}+\frac{1469.61114}{35}}=98.547 \mathrm{gram}
\end{gathered}
$$

And so can calculate the relative amplitude of the field of confidence on average through the following relationship:

$$
p=\frac{q}{\overline{\bar{X}}} \times 100=\frac{98.547}{796.8} \times 100=12.37 \%
$$

This means that the actual average of measurements in this test is located in the area:

$$
\overline{\bar{X}} \mp q=796.8 \mp 98.547 \text { gram }
$$


Results and proposals:

- The need to make sure shots clouds areas of capital annular spinning machines, in order to avoid a significant increase in the value of non-Evenness (which leads to a change number Twisting thus change tensile strength) and according to the effective length of the bristles operator.

- Ensure that the central rotation of the lower cylinder drag device and not a defect in the bearings hyphen between metal parts Cylinder Cylinders.ly top.

- The pressure must be applied its value specified on the upper cylinders, depending on the operating materials.

- Ensure the safety of the upper classes surfaces to prevent the occurrence of the accumulation of capillaries and they turn on their surface, which is bad for the quality of the thread operator.

- To ensure the validity of the count Traveler and type of raw material yarn type operators.

- Comply with the instructions of the manufacturer of Double companies, regarding the preliminary preparation for the new episodes of the program used for the first time (Running in Program).

- The need to ensure the safety of the metal surface of the rings to prevent affected Traveler rotational movement during the operational process.

- Replace Traveler according to the time periods described by its manufacturer companies.

- Development of the way while on the move, despite the presence of normal work by capillary cotton volatiles in the field to reduce the amount of friction between the Traveler and Episode (Traveler-Episode) and to ensure that a certain percentage reduction in friction.

- The study showed the need to find a way through which the cooling area of friction between Traveler and episodes without quality yarn operators affected (for example, the use of air cooling circuits or design Double or her self-cooling loops recipe), which is considered one of the most factors that lead to limited increase in velocities recently.

- It is necessary to develop a way to adjust the Traveler movement and reduce the lack of dynamic balance during the rotation around the central axis of the ring and Spindle.

- Balloon dimensions (length and bloating) resulting from Spindle and Traveler rotation, or change from one period to another, passing to a large extent on the nature of pulling the thread which is a primary indicator to determine the quality of the resulting string level, ridge to swell account, tells us there is a high strain wrap thread on pipe, and the high number Twisting and severity winding and high density wrap the pipe, and vice versa, and both cases are considered defects ring spinning, which means the need to look for ways to install the appropriate parameters affecting the value of pulling the thread.

We tried in this research study the effect of the string site (pipe top-pipe base) on the productivity and quality of yarn lumbar English count Ne 22 and using three different weights of the stitch and the study of their effect on the mechanical specifications and feel uniformity thread, the results showed an improvement in the properties of mechanical string of $\mathrm{Ne} 22$ in the top of the pipe compared while using Traveler with weight of $7.1 \mathrm{~g}$ per 100 pieces gives the best values for the feel, elongation, durability, necessary to cut off the power so these Traveler be suitable for spinning thread annular Ne 22 at speed spindle $14000 \mathrm{~d} / \mathrm{d}$.

Is the mechanical properties of the yarns of the basic parameters that describe the quality yarn and the mechanical properties are durability and tensile strength and elongation and the work required to cut. Virtually no property received more attention to them, most of the studies concerned with these characteristics have focused on the development of relations describing the mechanical properties of the yarns as a function of variables, structure and properties of the fiber [7].

In light of the differences and conflicting views of the quality of the thread should Ghazal to make thread and customized to suit the desired purpose. Can accomplish this through the unification of the quality of the thread to the general specifications of the final product, and this requires finding appropriate values of the properties of the thread and ideal machine. Quality thread translate into acceptable performance of the final product which is based on a clear and comprehensive overview of the production string to be consistent and reflecting both the requirements of the end user and this calls for a profound knowledge of the machine that affect the general thread characteristics variables, generally spinner knows the quality of the thread that:

- guide for superficial;

- tensile strength;

- evenness;

- defects level.

After all deer is more interested in seeing how the yarns users for their quality [6]. 
Where the ring spinning machine is one of the key species for the production of high quality yarn. The other spinning systems, production speed is higher than the annular spinning but restricted area of shortness of textile products and this is due to the limited technology. Basic technical opt-in ring spinning machine will be in the ring system-Traveler. Technology ring spinning machine is simple and old, but the achievement of quality and production requirements and put a lot of difficulties on the technical development of the machine in order to improve production efficiency and quality. The Traveler weight determines most of the frictional forces between them and the ring, and this in turn affects the rotation speed and tight balloon [3]-[7].

Can improve the quality of spinning choosing suitable for stitch weight, which leads to decrease thread, mass changes, changes, changes twisting and thus an increase in the quantity and quality of the product at a lower cost. Increase the speed of the machine is an important factor taking into account the age of the machine, there is always the desire to run the machine at high speeds, and this in turn leads to accelerate Traveler consumption, and increased cost due to the replacement of Traveler is a favorite against the increased productivity. Advised to use Traveler light yarns and high Traveler heavier yarn Thick, including Omar Traveler short it is necessary to replace them when needed and avoid unnecessary downtime of the machine. The appropriate choice of stitch determines experience and control the size of the balloon and the proportion of interruptions [8]-[10].

Winding through the spinning process, the strain at the beginning of the thread winding pipe is higher than at the end of the pipe wrap. This is because when the vehicle portion of the lower spray the thread tends to form a larger balloon due to air resistance [8] [9].

In this research was the study of the influence of floss site by testing three different weights to stitch and its impact on the productivity and quality of cotton yarn Ne 22 English and the speed of the machine cycle 14,000/d. The quality parameters that have been studied are thread, the thread tensile strength, elongation, the energy required to cut, thread rate per hour.

\section{The Aim of the Research}

Compared yarn specifications at the top of the ring spinning pipe and base the relationship with Traveler weight and hence study the impact of these two factors in tensile strength, elongation, the work required to cut, feel, uniformity leads. In order to reach the best conditions and variables run cotton yarn, so as to achieve a balance equation between the customer's requirements in terms of phenotypic cloth product while maintaining the mechanical properties in terms of durability threaded to the possibility of its use in weaving or knitting stage Least.

\section{Materials and Methods Used in the Research}

\subsection{Materials Used}

- Cotton yarn 100\% Ne 22 and average length of 30 (mm)—softness 5 (Maikaronner)—maturity of 85\%.

Yarn manufactured the machine spinning annular type Marazoli number spinners 960 spindles, was used operating variables as shown in Table 3.

\subsection{The Methods Used (Experimental)}

1) Test the mechanical properties of the yarns at the top of the pipe and test evenness and number twisting using three weights for stitch.

2) Test the mechanical properties of the yarns in the base of the pipe and test evenness and number twisting using three weights for stitch.

The experiment will be carried out on spinning Ne 22 as the tests that have been on the yarn before and after the application of the studied transactions is to measure each of:

1) Elongation and strength of the pieces, where all the experiments carried out on the tensile testing device, according to the following specifications:

Device model Testometric M350.

Jaws speed $500 \mathrm{~mm} / \mathrm{min}$.

The initial tensile $0.5 \mathrm{cN} / \mathrm{tex}$.

Speed the return of the jaws $500 \mathrm{~mm} / \mathrm{min}$.

The test sample length of $500 \mathrm{~mm}$.

2) Uniformity is a thread on evenness tester. 
3) Number twisting on Electronic Twist-Lab 2531c device.

Experiments conducted on ten samples (replicates) for each transaction, were taken to the arithmetic average of the measured values.

\section{TENSILE STRENGTH TEST:}

Table 4 shows Tensile strength testing results for spinning at the top and the base of the pipe under the influence of Traveler different weights.

Figure 12 shows the results compared to the power cut the thread at the top and the base of the pipe under the influence of different weights to stitch, where we note the following:

1) We note that the best values obtained were Traveler weight 7.1 (g) per 100 pieces in each of the summit and the base of the pipe.

2) Note the decline in these values in the pipe base compared with the top of the pipe, due to the fact that the tensile strength and the stress on the thread in the pipe than in the base peak.

\section{ELONGATION TEST:}

Table 5 shows results of the elongation of thread $(\mathrm{mm})$ at the top and the base of the pipe under the influence of Traveler different weights.

Figure 13 shows the results of elongation thread at the top and the base of the pipe under the influence of different weights to stitch and note the following:

1) We note that the yarn elongation decreases with increasing Traveler weight because the light weight of the stitch allows a number higher than twisting for one length for this reason elongation be higher when using stitch light and vice versa when using Traveler heavier.

2) We note the absence of a noticeable change elongation spinning at the top and the base of the pipe.

Table 3. Operating on the ring spinning machine variables.

\begin{tabular}{ccc}
\hline Yarn count (Ne) & Spindle (Rpm) & Traveler count ISO-Nr. \\
\hline 22 & 14000 & $90-71-58$ \\
\hline
\end{tabular}

Table 4. Results of tensile strength thread (CN) at the top and the base of the pipe under the influence of different weights of the stitch.

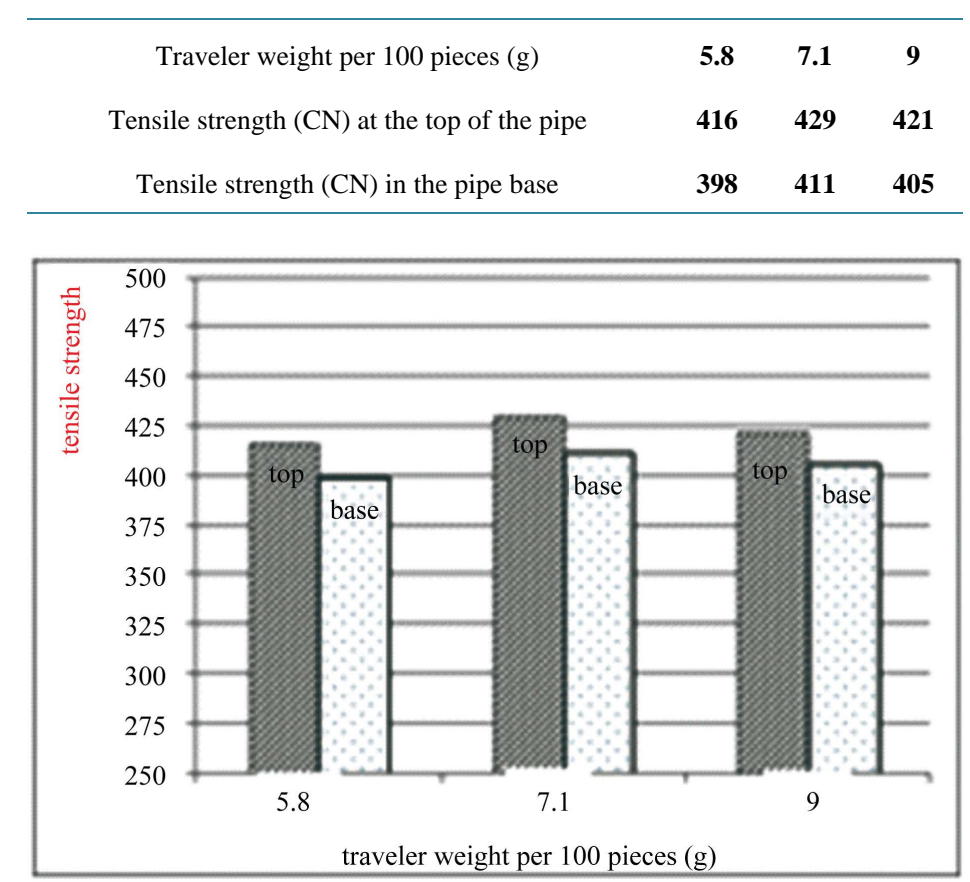

Figure 12. Comparison of tensile strength thread under the influence of different weights of the stitch. 


\section{TWISTING NUMBER TEST:}

Table 6 shows results of number of twisting (turns $/ \mathrm{m}$ ) at the top and the base of the pipe under the influence of Traveler different weights.

Figure 14 shows test number Twisting spinning at the top and the base of the pipe under the influence of different weights to stitch where we note the following results:

1) Traveler that weight has a clear impact on the number Twisting thread (turns $/ \mathrm{m}$ ) as the light weight of the stitch number Twisting gives up and this goes back to that Traveler be lighter surface speed higher than with heavier weight.

2) Comparing the results at the top and the base of the pipe note the absence of a noticeable change in the number of Twisting thread.

4. The impact of location on the pipe string and weight Traveler the uniformity and the level of defects in the leads:

The Table 7 shows test and the level of uniformity defects results in threaded at the top and the base of the pipe under the influence of different weights to stitch.

Figure 15 shows compare the results of the total defects spinning at the top and the base of the pipe impact of different weights to stitch where we note the following:

1) note when using Traveler of weight (7.1 g per 100 pieces) reduced the overall rate of defects while increasing the value of total public defects when using Traveler heavier and this is due to increased surface contact Traveler between Traveler thread and thus increase the stress on the capillaries passing through Traveler and thus increase the formation of defects regions.

Table 5. Results of the elongation of thread (mm) at the top and the base of the pipe under the influence of different weights of the stitch.

\begin{tabular}{cccc}
\hline Traveler weight per 100 pieces $(\mathrm{g})$ & $\mathbf{5 . 8}$ & $\mathbf{7 . 1}$ & $\mathbf{9}$ \\
elongation $(\mathrm{mm})$ at the top of the pipe & $\mathbf{3 0 . 6 1}$ & $\mathbf{3 0 . 1 4}$ & $\mathbf{2 7 . 8 3}$ \\
elongation $(\mathrm{mm})$ at the base of the pipe & 29.77 & $\mathbf{2 9 . 2 3}$ & $\mathbf{2 6 . 3 6}$ \\
\hline
\end{tabular}

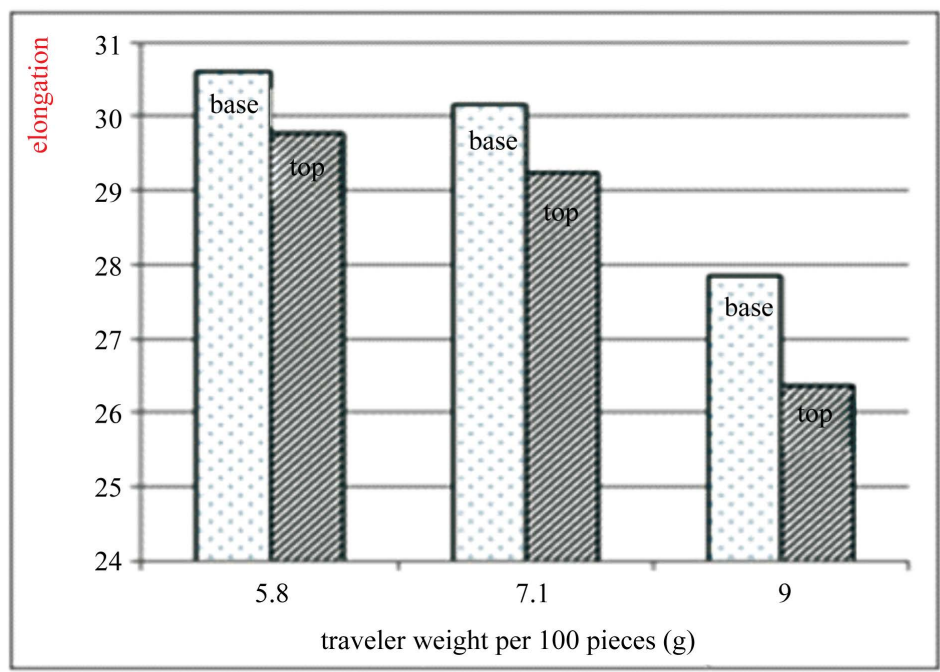

Figure 13. Comparison of elongation thread under the influence of different weights of the stitch.

Table 6. Shows the number of test results twisting (turns/m) at the top and the base of the pipe under the influence of Traveler different weights.

\begin{tabular}{cccc}
\hline Traveler weight per 100 pieces (g) & 5.8 & 7.1 & 9 \\
Number of twisting (turns/m) at the top of the pipe & 732 & 723 & 716 \\
Number of twisting (turns/m) at the base of the pipe & 723 & 717 & 711 \\
\hline
\end{tabular}




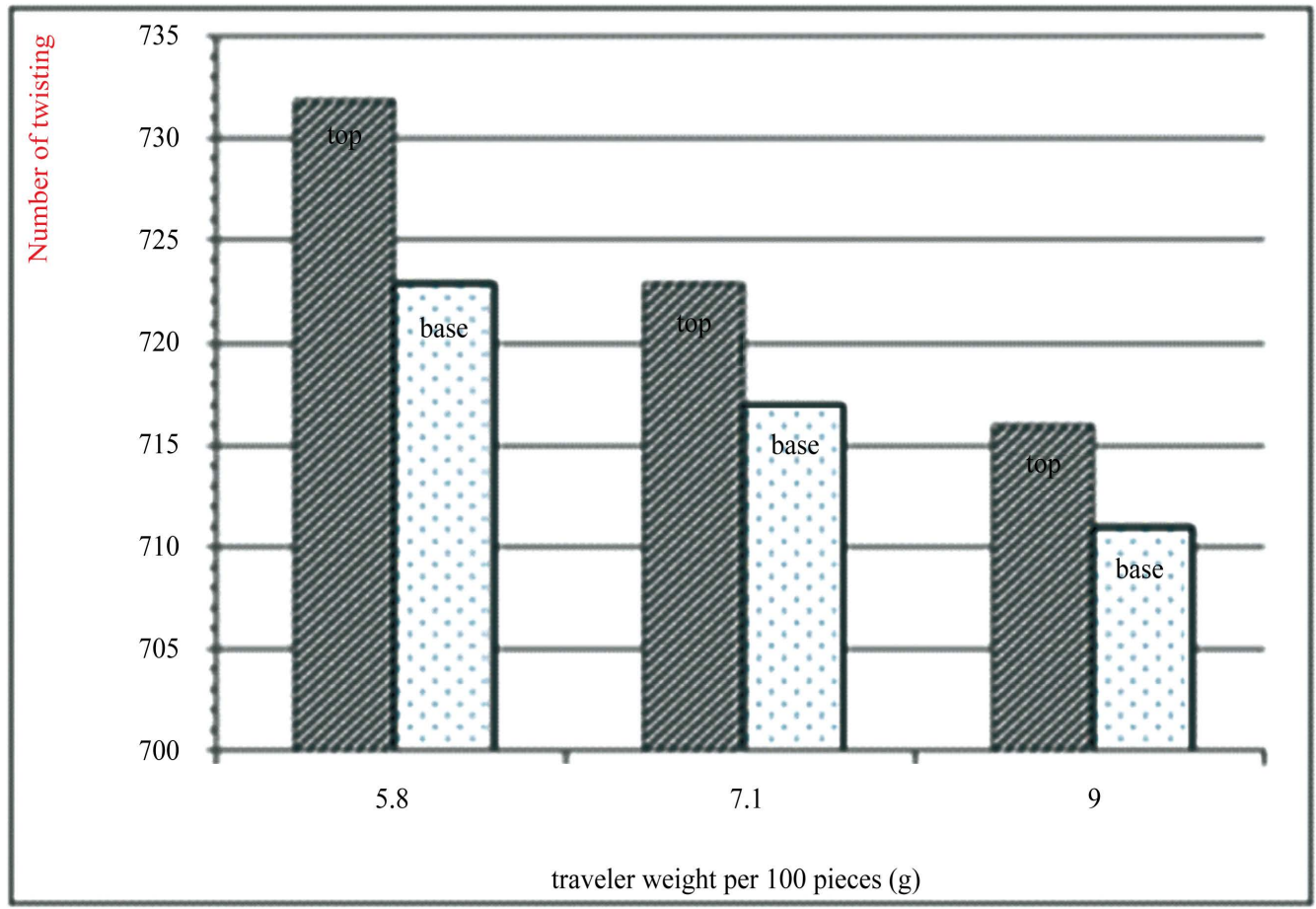

Figure 14. Comparison of the number of twisting spinning at the top and the base of the pipe under the influence of different weights to stitch.

Table 7. Values approval for weight Traveler per 100 pieces (g).

\begin{tabular}{|c|c|c|c|c|}
\hline & Traveler weight & 5.8 & 7.1 & 9 \\
\hline & Thick $+50 \% / \mathrm{km}$ & 642 & 327 & 1247 \\
\hline & Thick + 35\%/km & 2462 & 2125 & 3267 \\
\hline & Thin $-50 \% / \mathrm{km}$ & 29 & $\mathbf{0}$ & 59 \\
\hline \multirow[t]{7}{*}{ Pipe top } & Thin $-30 \% / \mathrm{km}$ & 2760 & 2854 & 4218 \\
\hline & Neps $+200 \% / k m$ & 671 & 520 & 720 \\
\hline & C.Vm \% & 17.29 & 16 & 19.46 \\
\hline & Total IPI & 1342 & 847 & 2026 \\
\hline & Thick $+50 \% / \mathrm{km}$ & 1195 & 985 & 1500 \\
\hline & Thick + 35\%/km & 2995 & 2770 & 3335 \\
\hline & Thin $-50 \% / \mathrm{km}$ & 35 & 65 & 100 \\
\hline \multirow[t]{4}{*}{ Pipe base } & Thin $-30 \% / \mathrm{km}$ & 4205 & 4250 & 5500 \\
\hline & Neps $+200 \% / k m$ & 965 & 840 & 940 \\
\hline & C.Vm \% & 19.27 & 19 & 20.4 \\
\hline & Total IPI & 2195 & 1890 & 2540 \\
\hline
\end{tabular}




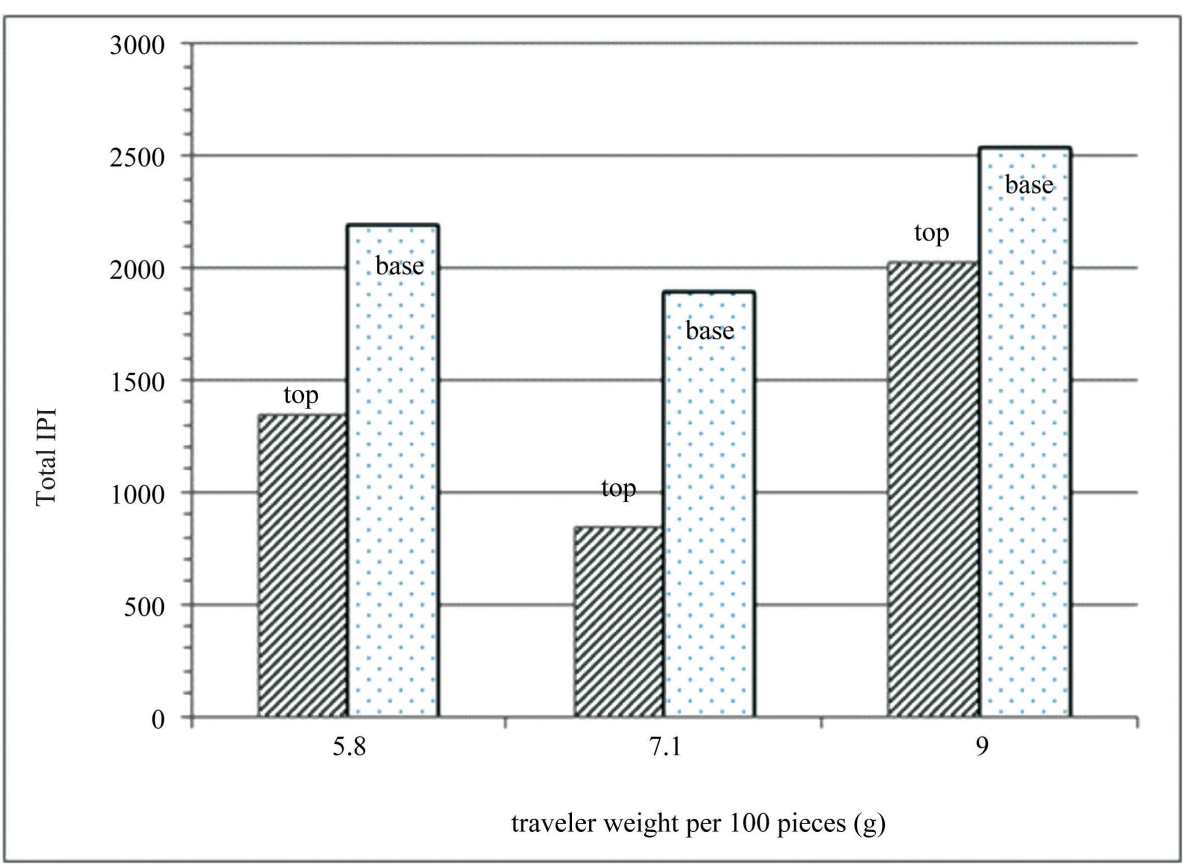

Figure 15. Comparison of the results of the total defects thread under the influence of different weights of the stitch.

2) increase the value of the total defects at the bottom of the barrel compared and was due to changes in tensile value associated with an increase in the balloon form the base of the pipe.

\section{Conclusions}

1) We note when using Traveler with weight of $7.1 \mathrm{~g}$ per 100 pieces, the thread gives the best values for the feel, elongation, durability, energy necessary to cut so these Traveler be suitable for spinning thread lumbar annular Ne 22 at speed Spindle 14,000 d/d.

2) We note that value in the base of the pipe is higher than the top of the pipe.

3) We also note that the mechanical properties of the thread at the top of the pipe to be better than the base of the pipe.

\section{Recommendations and Results}

1) We suggest choosing the appropriate Traveler because it gives the possibility to improve the production rate with the perfect specifications for quality.

2) In the ring spinning machines, the disadvantages resulting from the withdrawal of the previous stages of the devices will increase, and this is due to the following reasons:

- increased value difference in coefficient of linear density as a result of the lack of integration of operations during the checkout process;

- increased defects, according to the amount of drag applied to pull device for ring spinning machine;

- rotation central to spindle spinning machine because of the presence of corruption in;

- the existence of a defect in the clouds and the uncertainty of bullets related;

- differing impact on air balloon spinning all spindle result of irregular air density within the lounge;

- high and low vehicle during the lapping process.

3) Screwing in Traveler/manual thread is lower by almost 1.5 times tension in the string field (Traveler/pipe pulling the thread between the thread guide and cylinder supply), despite being lower than 2.5 times the tensile strength of the pipe and Traveler, but the effect is remarkable, given that it is weak incoherent applied to the material.

4) Laps less steadily guide thread; the balloon decreases and becomes more upright when Traveler increases 
even in the case of centrifugal force weak, and increasingly also tighten the thread area of friction between the ring and the framework.

\section{References}

[1] Salloum, W. (2010) Yarn Technology 1. Aleppo University Publications, Syria, 470 p. ( In Arabic)

[2] Lord, P.R. (2003) Handbook of Production. Technology, Science and Economics: Textile Institute, Woodhead Publishing Ltd., Cambridge. http://dx.doi.org/10.1201/9780203489710

[3] Lawrence, C.A. (2003) Fundamental of Spun Yarn Technology. Woodhead Publishing Ltd., Cambridge. http://dx.doi.org/10.1201/9780203009581

[4] Kameleo Makdecy, Dr.Eng. (2003) Fabric Tests. Al Dubs Press, Damascus.

[5] Mohammad Ahmad Sultan, Dr.Eng. (1992) Cotton Spinning Machines Mechanic. Al Intisar Press, Al Eskandaria.

[6] W. Schlafhorst \& Co. (1982) Information. Courtesy of W. Shlafhorst \& Co., 1-12.

[7] Howell, H.G. (1953) The General Case of Friction of a String Round a Cylinder. Journal of the Textile Institute Transactions, 8/9, T359-T362. http://dx.doi.org/10.1080/19447025308662594

[8] Klein, W. (1998) The Technology of Short Staple Spinning. Textile Institute, Manchester.

[9] Klein, W.A. (1987) Practical Guides to Combing \& Drawing and Spinning, Manuals of Textile Technology, 3 \& 4. Textile Institute, Manchester.

[10] Escher, R. (1883) Theory of the Ring Spindle. Der Civiingineur, 29,448

[11] De Barr, A.E. (1985) A Descriptive Account of Yarn Tensions and Balloon Shapes in Ring Spinning. Journal of the Textile Institute, 49, T58-T88. http://dx.doi.org/10.1080/19447025808660123 